\title{
Revisiting Sampson's theory for hydrodynamic transport in ultrathin nanopores
}

\author{
Mohammad Heiranian $\odot$, Amir Taqieddin $\odot$, and Narayana R. Aluru $\odot^{*}$ \\ Department of Mechanical Science and Engineering, University of Illinois at Urbana-Champaign, Urbana, Illinois 61801, USA
}

(Received 19 February 2020; accepted 1 October 2020; published 28 October 2020)

\begin{abstract}
Sampson's theory for hydrodynamic resistance across a zero-length orifice was developed over a century ago. Although a powerful theory for entrance/exit resistance in nanopores, it lacks accuracy for relatively small-radius pores since it does not account for the molecular interface chemistry. Here, Sampson's theory is revisited for the finite slippage and interfacial viscosity variation near the pore wall. The corrected Sampson's theory can accurately predict the hydrodynamic resistance from molecular dynamics simulations of ultrathin nanopores.
\end{abstract}

DOI: 10.1103/PhysRevResearch.2.043153

\section{INTRODUCTION}

Advances in experimentation [1-5] and computational studies [6-8] of fluids in nanoconfinement have led to the observation of unique properties of fluids. For example, water in carbon-based nanopores has been shown to have giant transport rates [5,8-11], fast rotational motion [12,13], and rotation-translation coupling [14]. Other solid-state nanopores such as transition metal dichalcogenides (TMDs) have also been explored and found to possess high water permeability and selectivity, making them attractive candidates for water desalination [15,16] and nanopower generation [17]. In addition, in biological nanopores, such as aquaporins, water exhibits a unique single-file dynamical behavior [18,19] with a high flux of about $10^{9}$ molecules per second due to the charge residues at the pore mouth causing water molecules to rotate and the conical structure of the pore minimizing the entrance/exit hydrodynamic resistance. In recent years, a great deal of attention has been given to two-dimensional (2D) materials (e.g., single-layer graphene, $\mathrm{MoS}_{2}$ ) due to their high transport rates $[10,15,20]$ as flux is expected (classically) to scale inversely with the thickness of the pore. For atomically thin pores, the flow rates are dictated by the entrance/exit hydrodynamic resistance governed by the viscous energy dissipation. The entrance/exit effects are also important in thicker pores where the resistance inside the pore is negligible $[21,22]$ [e.g., frictionless flows in carbon nanotubes (CNTs)]. In CNTs, accurate calculation of slip lengths, from experimentally measured permeation rates [9], depends on the accuracy of the theory used to obtain the entrance/exit resistance. As the entrance/exit resistance dominates the total resistance in a CNT, a slight error in the estimation of the entrance/exit resistance could lead to a large error in the

\footnotetext{
*aluru@illinois.edu

Published by the American Physical Society under the terms of the Creative Commons Attribution 4.0 International license. Further distribution of this work must maintain attribution to the author(s) and the published article's title, journal citation, and DOI.
}

calculation of slip length. Therefore, the need for an accurate theory describing the entrance/exit resistance (defined as the ratio of the pressure drop to the volumetric flow rate, $R=\frac{\Delta P}{Q}$ ) in both ultrathin and thick pores has been recognized in the past few decades [18,20,23].

Couette [24] in 1890 first introduced the entrance/exit resistance by crudely adding an additional length to the actual length of the tube in the Hagen-Poiseuille (HP) equation $[25,26]\left(R^{\mathrm{HP}}=\frac{8 \mu h}{\pi a^{4}}\right.$, where $\mu$ is the viscosity, $h$ is the tube length/thickness, and $a$ is the tube radius), which describes the flow in circular tubes independent of the end effects. A year later, Sampson [27] obtained the exact solution for the entrance/exit resistance of a zero-length orifice $\left(R^{\text {Sampson }}=\frac{3 \mu}{a^{3}}\right)$ within the framework of continuum fluid mechanics. The Sampson formula was later confirmed experimentally [28] for $\frac{h}{a}<26$. Weissberg [28] further found an upper bound for the entrance/exit resistance to be 1.154 times that of the Sampson formula using a variational method. Combining Sampson and HP equations, Dagan [29] obtained the total flow resistance across a finite-length orifice $\left[R^{\text {Dagan }}=\right.$ $\left.\frac{(8 h+3 \pi a) \mu}{\pi a^{4}}\right]$ using a no-slip boundary condition at the pore walls. More recently, hydrodynamic entrance/exit resistance was obtained for hourglass pores by modifying the prefactor in the Sampson formula based on finite-element calculations $[18,21]$. The Sampson formula, however, is found to overestimate the hydrodynamic resistance in graphene nanopores [21,23]. Gravelle et al. [21] attributed this overestimation to the finite thickness of a single-layer graphene nanopore. In this work, we show that the lower hydrodynamic resistance in graphene compared to that of the Sampson formula is due to the finite slippage at the edge of the pore. This is confirmed by modifying the molecular dynamics (MD) force-field parameters between water and graphene [resulting in a fictitious material having the same graphene structure but a different chemistry (highly hydrophilic)] such that a no-slip boundary condition is achieved at the pore edge. See Appendix A for details on the MD simulations performed in this study. Remarkably, the resistance obtained from MD simulations for the hydrophilic pore (with no slip) matches the resistance predicted by the Sampson formula. Motivated by this observation in MD simulations, we revisit the Sampson 

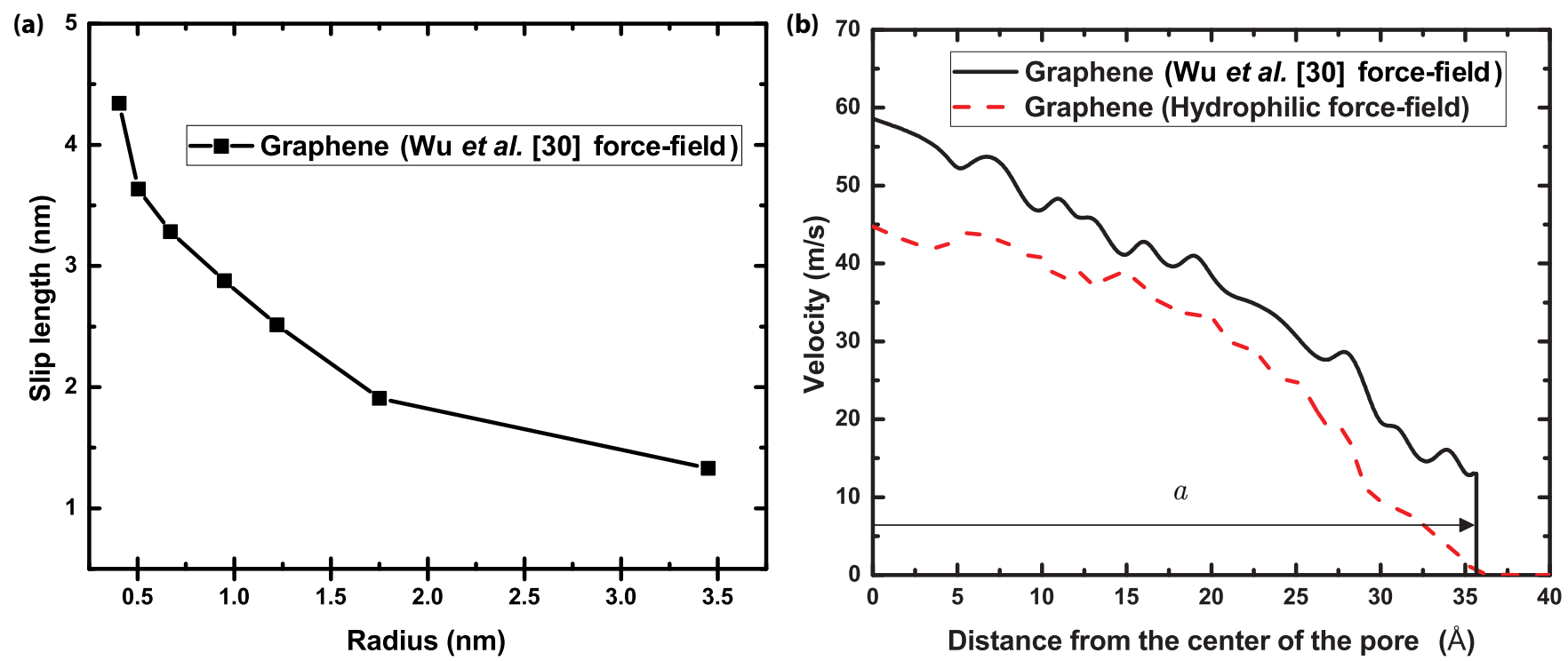

FIG. 1. (a) The slip lengths in the graphene nanopore are plotted for different radius $(a)$. Finite slip lengths are obtained when using the Wu et al. [30] force field. (b) Axial velocity profile in the single-layer graphene nanopore $(a=3.54 \mathrm{~nm})$ as a function of the radial distance from the center of the pore is plotted using the Wu et al. [30] force field (black solid curve) and a fictitious hydrophilic force field $\left(\varepsilon_{\mathrm{C}-\mathrm{O}}=0.9 \mathrm{kcal} / \mathrm{mol}\right.$, red dashed curve [see Appendix A (Table I) and B (Fig. 8)]). The water accessible radius $a$ is illustrated by an arrow. The water accessible radius is the length within which the water density is nonzero [see Appendix B (Fig. 8(c))]. In Appendix G, a systematic method of calculating the accessible radius is discussed.

theory and introduce a finite slip length $(\delta)$ into the original theory. Introducing a finite slip length for a nearly zero-length orifice in Sampson's theory may be counterintuitive at first; however, a slip length can be introduced if the velocity at any cross section is known, e.g., the velocity profile right at the orifice. The deviation of the resistance in MD simulations from that of the original Sampson theory is found to be dictated by the ratio of slip length to radius, $\frac{\delta}{a}$. To further account for the interfacial molecular physics, we corrected the Sampson theory for the variation of viscosity at the interface of the graphene membrane. The slip- and viscosity-corrected Sampson's theory is able to predict the hydrodynamic resistance from MD simulations for different values of $\frac{\delta}{a}$ (which represents a measure of both the geometry and chemistry of the pore) with no adjustable parameters. In addition to MD simulations, steady-state Navier-Stokes (NS) continuum simulations are carried out where the hydrodynamic resistance matches the values predicted by the corrected Sampson formula (see Appendix C for more details on NS simulations).

\section{ROLE OF SLIPPAGE}

Water transport in single-layer graphene nanopores is associated with slippage at the pore due to the significance of molecular physics between water molecules and carbon atoms. Suk et al. [10] investigated water transport in graphene nanopores where a parabolalike velocity profile with a slip velocity at the interface was observed. The slip lengths in graphene are one order of magnitude smaller than those of infinitely long CNTs where pluglike velocity profiles are observed [8]. Slip length in graphene decreases with an increase in radius as shown in Fig. 1(a) (see Appendix A for details on slip calculation). The slip length variation is explained [23] by the decrease in viscosity and increase in the interfacial friction coefficient as radius increases. In the original Sampson theory, a no-slip condition is assumed at the wall and an elliptical velocity profile is obtained inside the pore. Slippage in graphene results in higher permeability (or lower resistance) compared to the permeability predicted by the original Sampson theory. In this work, the interaction parameter between the oxygen atom of water and the carbon atom of graphene is changed such that a no-slip interface (hydrophilic wall) is achieved in MD simulations [see Appendix A (Table I) and Fig. 8]. In Fig. 1(b), the axial velocity profile of the fictitious hydrophilic pore is compared to that of the nonfictitious graphene (Wu et al. [30] force field) for a radius of $3.54 \mathrm{~nm}$. The hydrodynamic resistance is directly computed from MD simulations to be $5.78 \pm 0.03 \times 10^{22} \mathrm{~Pa} \mathrm{~s} / \mathrm{m}^{3}$ and $4.21 \pm$ $0.4 \times 10^{22} \mathrm{~Pa} \mathrm{~s} / \mathrm{m}^{3}$, for the hydrophilic pore and graphene pore, respectively. The resistance obtained for the hydrophilic pore matches the resistance predicted by the Sampson theory $\left(R^{\text {Sampson }}=\frac{3 \mu}{a^{3}}=5.75 \times 10^{22} \mathrm{~Pa} \mathrm{~s} / \mathrm{m}^{3}\right.$, where $\mu$ is taken to be the bulk viscosity of water $(0.85 \mathrm{mPas})$ and $a$ is calculated based on the water accessible radius [see Fig. 1(b)]). Sampson theory, however, fails to predict the resistance for the graphene nanopore (where a slip length exists) as it does not include interfacial physics in the form of slip length and viscosity variation. Motivated by the results in Fig. 1, the effect of slip length is introduced into Sampson's theory in the next section.

\section{SLIP-CORRECTED SAMPSON'S THEORY}

The pressure drop, $\Delta P$, can be obtained from the rate of energy dissipation using [31]

$$
\Delta P=\frac{2 \mu}{Q} \int \omega^{2} d V+\frac{4 \mu}{Q} \int(\mathbf{u} \cdot \nabla) \mathbf{u} \cdot \mathbf{n} d S,
$$

where $\mu$ is the fluid viscosity, $Q$ is the volumetric flow rate, $\omega$ is the vorticity, $V$ is the volume, $\mathbf{u}$ is the flow field, and $S$ is the surface of the boundaries of the system. For simplification, the 
(a)

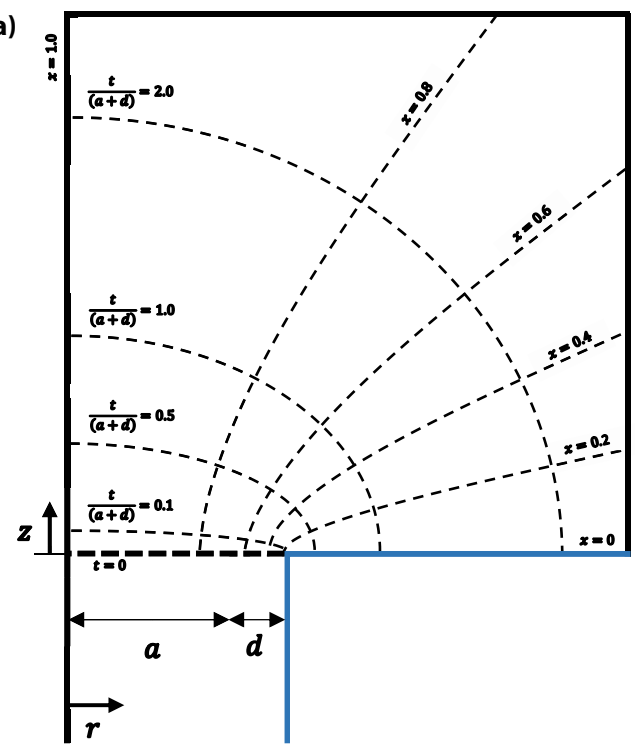

(b)

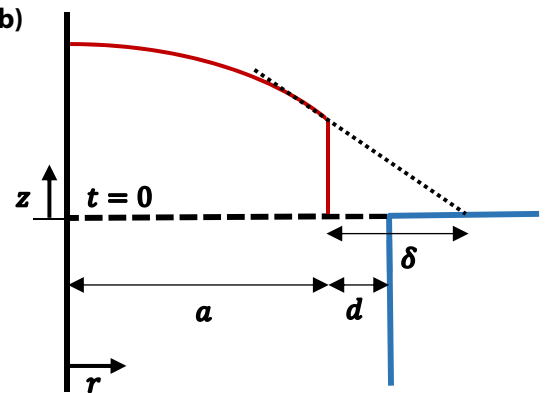

FIG. 2. (a) The confocal hyperbolas and ellipses (denoted by $x$ and $t$, respectively) in the oblate spheroidal coordinates are shown for an orifice with a water accessible radius of $a$. The orifice boundaries are presented in blue. Inside the orifice, the radial distance from the center of the orifice (denoted by $r$ ) is shown by a solid arrow. The oblate spherical coordinates (outside the orifice) and the cylindrical coordinates (inside the orifice) are related by Eqs. (2) and (3). (b) The schematic of the velocity profile (red curve) and the lengths considered in the Sampson formulation are shown. The dotted line is the tangent line to the velocity profile at $r=a$ whose intersection with $z=0$ defines the slip length $\delta . d$ is a virtual length within which the velocity is zero. Note that the cylindrical sections of the two schematics have finite thicknesses (the actual pore thickness is zero in the theory) merely for the sake of clear presentation.

second term in Eq. (1) can be neglected assuming the velocity on the boundaries of $S$ to be zero. In this work, ultrathin pores are represented by a zero-length orifice. Oblate spheroidal coordinates are used outside the orifice and cylindrical coordinates inside the orifice as shown in Fig. 2(a). $x$ and $t$ in the oblate spheroidal coordinates are related to the cylindrical coordinates $z$ and $r$ using

$$
\begin{aligned}
& r^{2}=\left[t^{2}+(a+d)^{2}\right]\left(1-x^{2}\right), \\
& z^{2}=t^{2} x^{2}
\end{aligned}
$$

where $d$ is the virtual length, characterizing the slip length as discussed later in more detail, in the radial direction of the cylindrical coordinates. The vorticity in Eq. (1) can be expressed in terms of the stream function $\psi$ using [32]

$$
r \omega=-D^{2} \psi,
$$

where $D^{2}$ is the differential operator (see Appendix E for the definition of $D^{2}$ ). Using Eqs. (1)-(4), the pressure drop can be rewritten as

$$
\begin{aligned}
\Delta P= & \frac{4 \pi \mu}{Q} \int_{0}^{1} \int_{0}^{\infty} \frac{1}{t^{2}+(a+d)^{2} x^{2}}\left\{\left[\frac{t^{2}+(a+d)^{2}}{1-x^{2}}\right]^{\frac{1}{2}}\right. \\
& \left.\times \frac{\partial^{2} \psi}{\partial t^{2}}+\left[\frac{1-x^{2}}{t^{2}+(a+d)^{2}}\right]^{\frac{1}{2}} \frac{\partial^{2} \psi}{\partial x^{2}}\right\}^{2} d t d x .
\end{aligned}
$$

The exact stream function, $\psi^{o}=\frac{1}{2 \pi} Q x^{3}$, which Sampson [27] obtained for flow through a zero-length orifice, when used in Eq. (5) results in the famous Sampson formula, $\Delta P=\frac{3 \mu Q}{a^{3}}$. A trial stream function [Eq. (6)] is used that best mimics the flow fields in the simulations (a velocity jump at the depletion region boundary and an almost vanishing velocity near the outside walls [see Appendix D, Fig. 10]) by setting the stream function (or velocity) to zero from $0<x \leqslant b$ :

$$
\psi= \begin{cases}\frac{1}{2 \pi} Q x^{3} & \text { for } b<x \leqslant 1, \\ 0 & \text { for } 0<x \leqslant b,\end{cases}
$$

where $b$ in the oblate spheroidal coordinates corresponds to $d$ [the virtual length within which the velocity is set to zero as shown in Fig. 2(b)] in the cylindrical coordinates when $r=a$ at the edge of the pore $(t=0)$. The stream function in (6) satisfies the boundary conditions used in the original Sampson theory $\left[U_{t}=0\right.$ and $U_{x}=0$ as $t \rightarrow \infty, U_{t}=0$ and $U_{x}=0$ at $x=0$ (outside the orifice tube), and $U_{r}=0$ and $U_{\phi}=0$ at $r=a+d$ (inside the orifice tube where $t=0$ )]. $\phi$ is the angular coordinate inside the orifice tube. Using the trial stream function, the streamline that corresponds to the slip velocity inside the pore smoothly varies across the pore as shown in Appendix D, Fig. 10(a) (the dashed curve at $x=b$ ). Therefore, the velocity direction at the vertex point, where we have the sharp pore corners, is smoothly varying. The stream function in Eq. (6), however, results in a velocity discontinuity at $x=b$. This discontinuity can be eliminated by considering, for example, another trial stream function (see Appendix F for more details) which leads to an almost similar hydrodynamic resistance as that obtained from the stream function in Eq. (6). Using Eq. (2), $b$ can be expressed in terms of the virtual length $d$ as follows:

$$
b=\left(\frac{d}{a+d}\right)\left(1+\frac{2 a}{d}\right)^{\frac{1}{2}} .
$$

Slip length is related to the slip velocity, which is the $t$ component of velocity at $r=a$, using the relation $U_{\text {slip }}=\delta \frac{\partial U_{t}}{\partial r}$ at the 
pore $\left[U_{t}(t=0\right.$ and $\left.r=a)=U_{\text {slip }}\right] . U_{t}$ can be obtained from the stream function $\psi$ using

$$
U_{t}=\frac{1}{r h_{x}} \frac{\partial \psi}{\partial x}=\frac{3 Q}{2 \pi} \frac{x^{2}}{\left[t^{2}+(a+d)^{2}\right]^{1 / 2}\left[t^{2}+(a+d)^{2} x^{2}\right]^{1 / 2}},
$$

where $h_{x}$ is the scale factor in the oblate spheroidal coordinates (see Appendix E for the definition of the scale factors $h_{x}, h_{t}$, and $h_{\phi}$ ). The slip length $\delta$ (the ratio of $U_{t}$ to $\frac{\partial U_{t}}{\partial r}$ at $t=0$ and $r=a$ ) can then be expressed in terms of the virtual length $d$ as

$$
\delta=\frac{d^{2}+2 a d}{a} .
$$

In Eq. (9), for a given radius, the slip length is obtained corresponding to a value of $d$. Now using Eqs. (6), (7), and (9), the pressure drop in Eq. (5) can be expressed in terms of the ratio of slip length to radius $\left(\alpha=\frac{\delta}{a}\right)$. The Sampson formula corrected for slippage is, therefore, given by

$$
R_{\text {Slip-corrected }}^{\text {Sampson }}=\frac{\Delta P}{Q}=\frac{3 \mu}{a^{3}}\left[\frac{1+2\left(\frac{\alpha}{1+\alpha}\right)^{3 / 2}-3\left(\frac{\alpha}{1+\alpha}\right)}{(1+\alpha)^{3 / 2}}\right] .
$$

As $\alpha \rightarrow 0(\delta \rightarrow 0$ or $a \rightarrow \infty)$, when there is no slippage or the radius is very large compared to the slip length, the hydrodynamic resistance in Eq. (10) reduces to the original Sampson resistance $\left(R_{\text {Slip-corrected }}^{\text {Sampson }} \rightarrow R^{\text {Sampson }}=\frac{\Delta P}{Q}=\frac{3 \mu}{a^{3}}\right)$. The hydrodynamic resistance predicted by the slip-corrected Sampson formula normalized by that of the original Sampson formula $\left(\frac{R_{\text {Sipp-conrected }}^{\text {Samson }}}{R^{\text {Sampson }}}=\left[\frac{1+2\left(\frac{\alpha}{1+\alpha}\right)^{3 / 2}-3\left(\frac{\alpha}{1+\alpha}\right)}{(1+\alpha)^{3 / 2}}\right]\right)$ as a function of the ratio of radius to slip length $\left(\frac{1}{\alpha}=\frac{a}{\delta}\right)$ is plotted in Fig. 3. The slip-corrected Sampson's theory is able to match the hydrodynamic resistance from MD and NS simulations (used as the reference methods) for all the graphene pore radii and water-carbon interaction parameters (which characterize the interface chemistry or the slip length) except for small values of $\frac{a}{\delta}<1$. Tables I and II summarize all the MD and NS simulations performed in this study. The original Sampson's theory, however, fails to predict the hydrodynamic resistance from MD simulations for $\frac{a}{\delta}<300$.

\section{VISCOSITY-CORRECTED SAMPSON'S THEORY}

The slip-corrected Sampson formula underestimates the hydrodynamic resistance for small values of $\frac{a}{\delta}<1$ as it does not account for the variation of the interfacial viscosity at the membrane in the MD simulations. It is known that the viscosity of water at graphitic interfaces reduces (due to the higher than bulk diffusion) and enhances (due to the highly layered structure of water) for the flow parallel and perpendicular to the plane of the interface, respectively $[23,33,34]$. The average effective interfacial shear viscosity (within the interface layer with a thickness of $L_{\mathrm{int}}=0.8 \mathrm{~nm}$ ) is calculated in MD simulations using the Green-Kubo formulation (refer to Ref. [23] for more details) to be $\mu_{\text {int }}=0.98 \mathrm{mPa}$ s. $L_{\text {int }}$ is the distance from the graphene wall within which the viscosity variation takes place as shown in Fig. 4. To find the thickness of the interfacial layer, a separate MD simulation is carried out [Fig. 4(a); a flat graphene membrane (no pores) with water molecules (containing $\sim 118000$ atoms with dimensions of $16.3 \mathrm{~nm} \times 3 \mathrm{~nm} \times 3 \mathrm{~nm}$ )]. In Fig. 4(b),

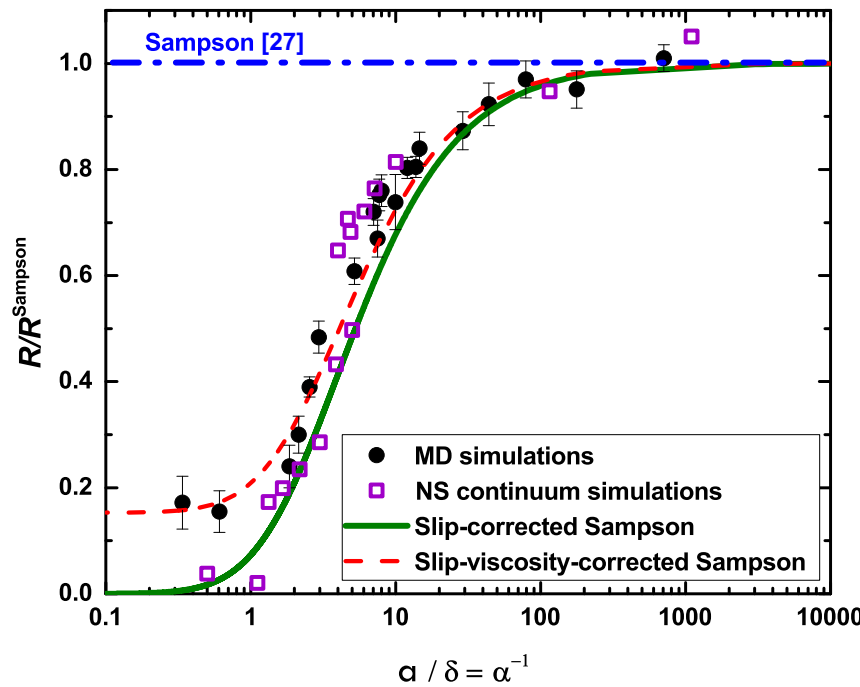

FIG. 3. Comparison of the hydrodynamic resistance $(R)$ obtained from MD simulations, NS continuum simulations, and the corrected Sampson's theory to that of the original Sampson's theory (blue dash-dotted line) for different radius-to-slip length ratios $\left(\frac{1}{\alpha}=\frac{a}{\delta}\right)$. For smaller ratios (small radii or large slip lengths), the original Sampson formula overestimates the resistance from MD simulations (black solid symbols). The slip-corrected Sampson formula [green solid curve, Eq. (10)] describes the flow quite well from NS simulations (purple hollow symbols) as well as MD simulations. The inclusion of viscosity correction, in addition to the slippage [red dashed curve, Eq. (14)] near the graphene interface leads to a good match with MD simulations [as viscosity variation take places in MD simulations (in NS simulations, the viscosity is assumed to be uniform)].

considering all the shear components of the stress tensor, the average viscosity $\left(\mu_{\text {ave }}=\frac{\mu_{12}+\mu_{13}+\mu_{23}}{3}\right)$ is calculated in different slabs (parallel to the graphene sheet) with a thickness of $0.3 \mathrm{~nm}$ each (the black symbols). As shown, most of the variation takes place within $\sim 0.8 \mathrm{~nm}$ of the graphene wall. Therefore, $L_{\text {int }}$ is set to be $0.8 \mathrm{~nm}$ and the average effective interfacial viscosity is calculated to be $\mu_{\text {int }}=0.98 \mathrm{mPa}$ s. To obtain the pressure drop from Eq. (5), the integration must be carried out such that the viscosity is $\mu_{\text {int }}=0.98 \mathrm{mPa} \mathrm{s}$ for $0<z \leqslant L_{\text {int }}$ and $\mu_{\text {bulk }}=0.85 \mathrm{mPa}$ s, elsewhere. The interval $0<z \leqslant L_{\text {int }}$ corresponds to $0<t \leqslant \frac{L_{\text {int }}}{x}$ in the oblate spheroidal coordinates using Eq. (3). Therefore, Eq. (5) can be rewritten as

$$
\begin{aligned}
\Delta P & =\frac{4 \pi}{Q} \int_{0}^{1} \int_{0}^{\infty} \mu(x, t) f(x, t) d t d x \\
& =\frac{4 \pi}{Q} \int_{0}^{1}\left[\int_{0}^{\frac{L_{\text {int }}}{x}} \mu_{\text {int }} f(x, t) d t+\int_{\frac{L_{\text {int }}}{x}}^{\infty} \mu_{\text {bulk }} f(x, t) d t\right] d x,
\end{aligned}
$$

where $f(x, t)$ is the integrand in Eq. (5). The interfacial viscosity can be expressed in terms of the bulk viscosity and an excess viscosity $\left(\mu_{\text {int }}=\mu_{\text {excess }}+\mu_{\text {bulk }}\right.$ where $\mu_{\text {excess }}=0.13 \mathrm{mPas}$ for graphene). The pressure drop in Eq. (11) can be rewritten in terms of the excess and bulk 

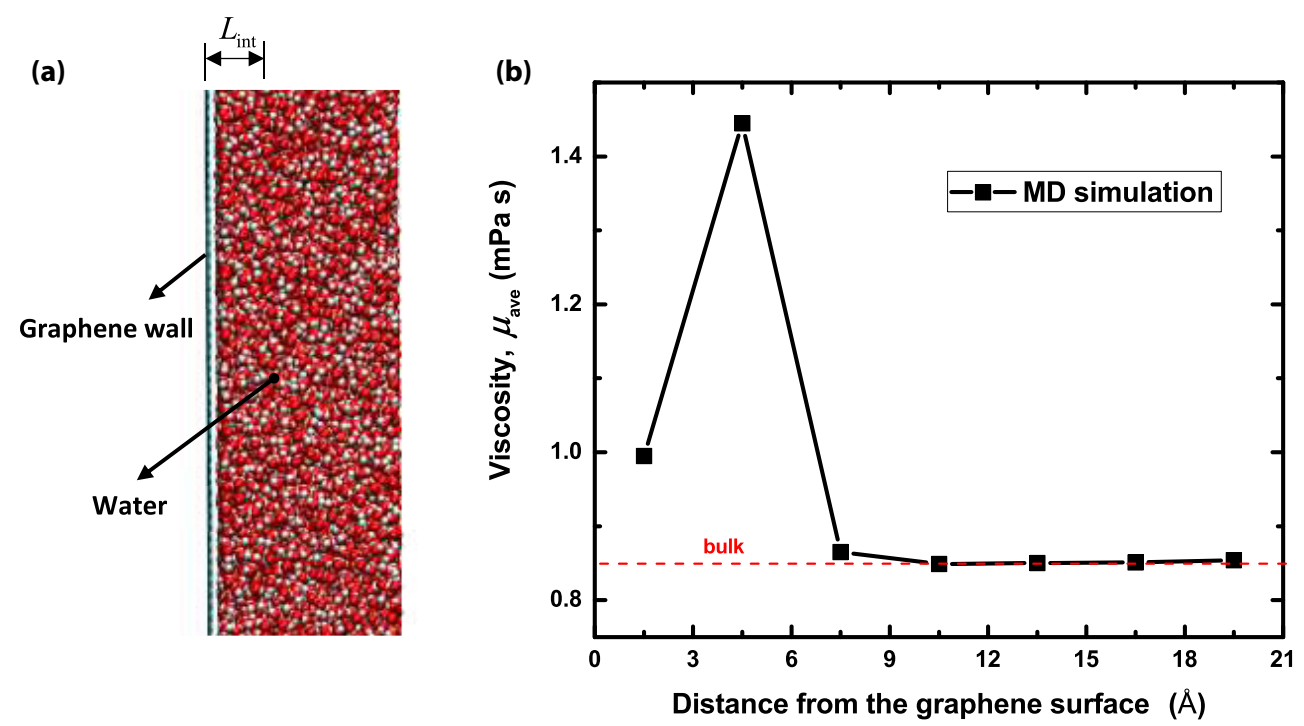

FIG. 4. (a) Simulation box for water viscosity calculation near a water-graphene interface. Oxygen, hydrogen, and carbon atoms are shown in red, white, and cyan, respectively. The interfacial layer $\left(L_{\text {int }}\right)$ within which viscosity varies is shown. (b) Viscosity as a function of the distance from the graphene surface is shown (black symbols). The bulk viscosity ( $\mu_{\text {bulk }}=0.85 \mathrm{mPa}$ ) is indicated by the dashed red line. For the viscosity-corrected curve in Fig. $3, \mu_{\text {excess }}=0.13 \mathrm{mPa} \mathrm{s}, L_{\text {int }}=0.8 \mathrm{~nm}$, and a constant slip length of $\delta=1.5 \mathrm{~nm}$ are assumed.

viscosities as

$$
\begin{aligned}
\Delta P= & \frac{4 \pi \mu_{\mathrm{excess}}}{Q} \int_{0}^{1} \int_{0}^{\frac{L_{\text {int }}}{x}} f(x, t) d t d x+\frac{4 \pi \mu_{\text {bulk }}}{Q} \\
& \times \int_{0}^{1} \int_{0}^{\infty} f(x, t) d t d x,
\end{aligned}
$$

where the second term is simply the pressure drop in Eq. (5). In other words, the total pressure drop in Eq. (12) is the sum of the pressure drop across the orifice with bulk viscosity and an additional pressure drop due to the excess viscosity at the interface. Therefore, the total hydrodynamic resistance can be given by

$$
R_{\text {Slip-viscosity-corrected }}^{\text {Sampson }}=R_{\text {Slip-corrected }}^{\text {Sampson }}+R_{\text {excess-viscosity }}^{\text {Sampson }},
$$

where $R_{\text {Slip-corrected }}^{\text {Sampson }}$ is the slip-corrected Sampson formula in Eq. (10) and the hydrodynamic resistance due to the excess viscosity can be obtained from integrating the first term in Eq. (12) (the effect of slippage for the viscosity-corrected term is neglected for simplicity) as follows:

$$
R_{\text {excess-viscosity }}^{\text {Sampson }}=\frac{6 \mu_{\text {excess }}}{\pi a^{3}}\left[\tan ^{-1}(\beta)+\ln \left(\frac{1}{\beta^{2}}+1\right)\left(\frac{3 \beta}{2}+\beta^{3}\right)-\beta\right],
$$

where $\beta$ is the ratio of the interfacial layer thickness to radius $\left(\beta=\frac{L_{\text {int }}}{a}\right)$. In Fig. 3, the hydrodynamic resistance predicted by the slip- and viscosity-corrected Sampson formula normalized by that of the original Sampson formula as a function of the ratio of the radius of the pore to the slip length is compared with the MD simulations. The slip- and viscosity-corrected Sampson's theory gives rise to a good match with the hydrodynamic resistance from the MD simulations even for small values of $\frac{a}{\delta}$ where the slip-corrected Sampson's theory fails. The additional resistance due to the excess interfacial viscosity is dictated by $\beta=\frac{L_{\text {int }}}{a}$. In Fig. 5 , the normalized additional resistance is plotted as a function of $\beta$. As $\beta \rightarrow 0$ ( $L_{\text {int }} \rightarrow 0$ or $a \rightarrow \infty$ ), when the interfacial viscosity variation length is negligible or the radius is very large compared to the interfacial layer thickness, the excess viscosity hydrodynamic resistance approaches zero $\left(R_{\text {excess-viscosity }}^{\text {Sampson }} \rightarrow 0\right)$. The normalized $R_{\text {excess-viscosity }}^{\text {Sampso }}$ reaches a plateau beyond a critical $\beta$ for which most of the hydrodynamic pressure drop takes place within $L_{\text {int }}$. In other words, $L_{\text {int }}$ becomes comparable to the hydrodynamic pressure drop length. In Dagan's theory [29], the effective length over which the pressure drop takes place is given by $h^{\text {Dagan }}=h+\frac{3}{8} \pi a$. For a zero-length orifice, as $h \rightarrow 0$, the ratio of the hydrodynamic pressure drop length to radius is found to be $\frac{h^{\text {Dagan }}}{a}=\frac{3}{8} \pi=1.178$, which exactly corresponds to the critical value of $\beta$ at which the excess viscosity hydrodynamic resistance reaches a plateau.

\section{FINAL REMARKS}

Sampson's theory has been recently used to describe the flow in ultrathin pores and to understand the entrance/exit hydrodynamic resistance in nanotubes. Although a powerful theory, it fails to predict the flow in small-diameter graphene nanopores. Here, we show that the pore slippage, which is not accounted for in the original Sampson theory, plays an important role. The original Sampson theory is revisited and 


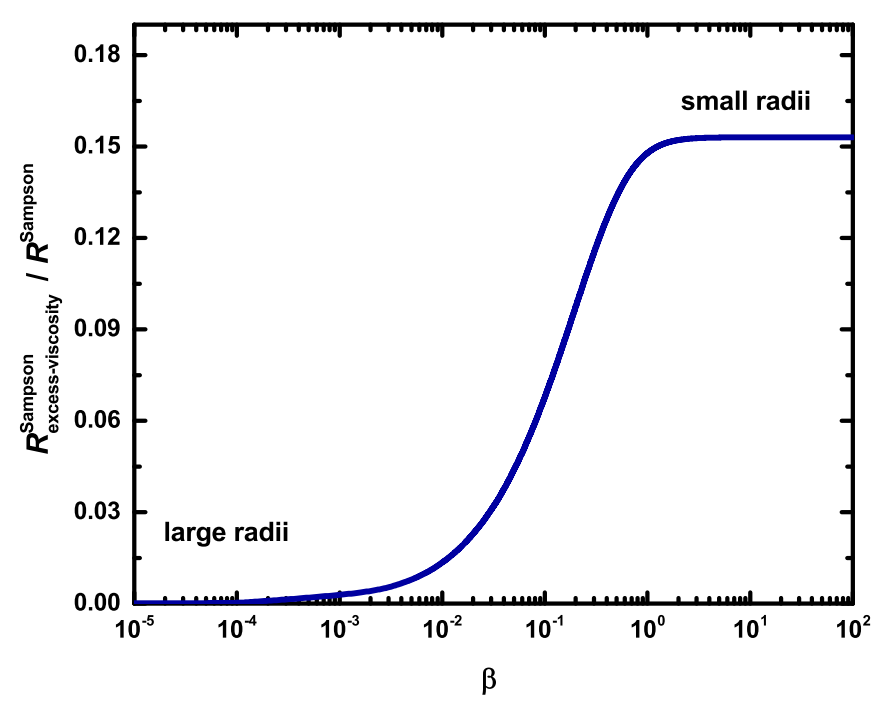

FIG. 5. Hydrodynamic resistance due to the excess interfacial viscosity [Eq. (14)] normalized by the hydrodynamic resistance of the original Sampson theory is plotted as a function of the ratio of the interface layer thickness to radius, $\beta=\frac{L_{\text {int }}}{a}$. In Eq. (14), $\mu_{\text {excess }}=$ $0.13 \mathrm{mPa}$ s and $L_{\text {int }}=0.8 \mathrm{~nm}$ are obtained from MD simulations. For small $\beta$ (negligible interfacial viscosity variation or large radii), the excess hydrodynamic resistance is insignificant. For large $\beta$ (when it is comparable to the ratio of the effective hydrodynamic pressure drop length to radius), the contribution of the excess resistance to the total resistance becomes important (as shown for small $\frac{a}{\delta}$ in Fig. 3).

corrected for the slippage and the variation of viscosity at the membrane interface. We introduced a set of formulas for the slip- and viscosity-corrected Sampson's theory which results in good agreement with data from MD and NS simulations as well as experiments. The permeation coefficients predicted using the corrected Sampson theory are compared to the experimentally measured permeations in single-layer graphene nanopores in Fig. 6(a). The discrepancy between the permeation coefficients obtained from the experiments and the Sampson theory are possibly due to (i) lack of accurate force fields for graphene nanopores where quantum and other effects could be important (as MD simulations are used as the reference model for calculating slip lengths), (ii) challenging flow rate measurements and existence of defects and contaminants in experiments, and (iii) inaccurate measurement of pore radii in experiments (a slight change in the value of the radius results in a large change in the permeation coefficient predicted by the Sampson theory, etc.). The ratio of slip length to the radius of the pore is the key parameter in explaining the flow in ultrathin nanopores. Since the slip length depends on the chemistry of the membrane, our theory is applicable to any 2D material or ultrathin pore (e.g., pores in graphene, TMDs, boron nitride, phosphorene). With the recent interest in nanoporous 2D materials having potential applications in water desalination [15,35], energy generation [17], and healthcare [36,37], there is a need for more accurate theories such as the one developed here. Note that the model presented here ideally works for circular pores in 2D materials (see Appendix $\mathrm{H}$ ). We should also note that our model does account for the effect of the possible charge distributions at the pores on the hydrodynamic flow. Aside from porous 2D materials and other porous ultrathin membranes, accurate estimation of entrance/exit hydrodynamic resistance is required in frictionless nanotubes (e.g., CNTs) (a)

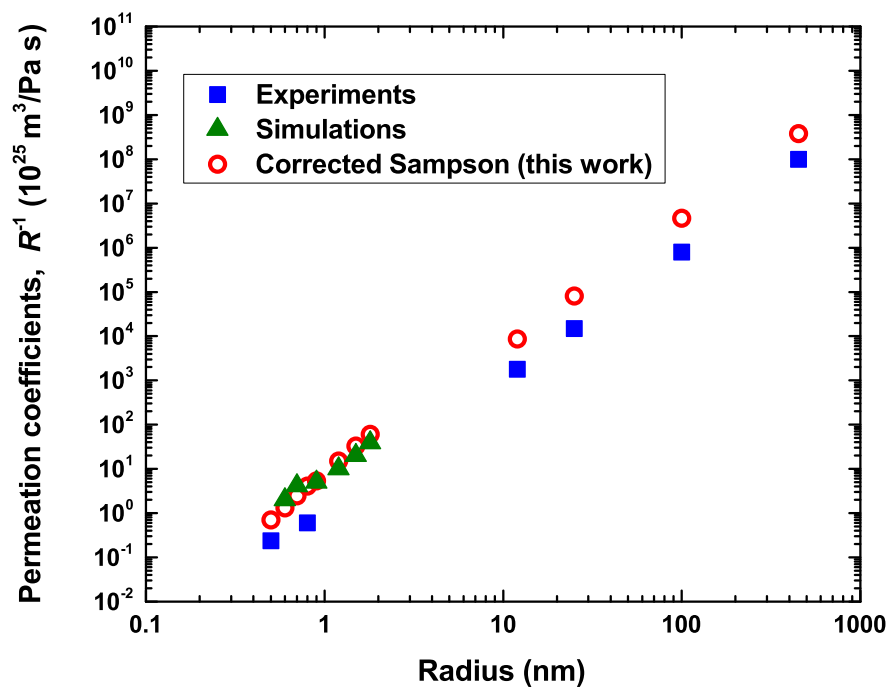

(b)

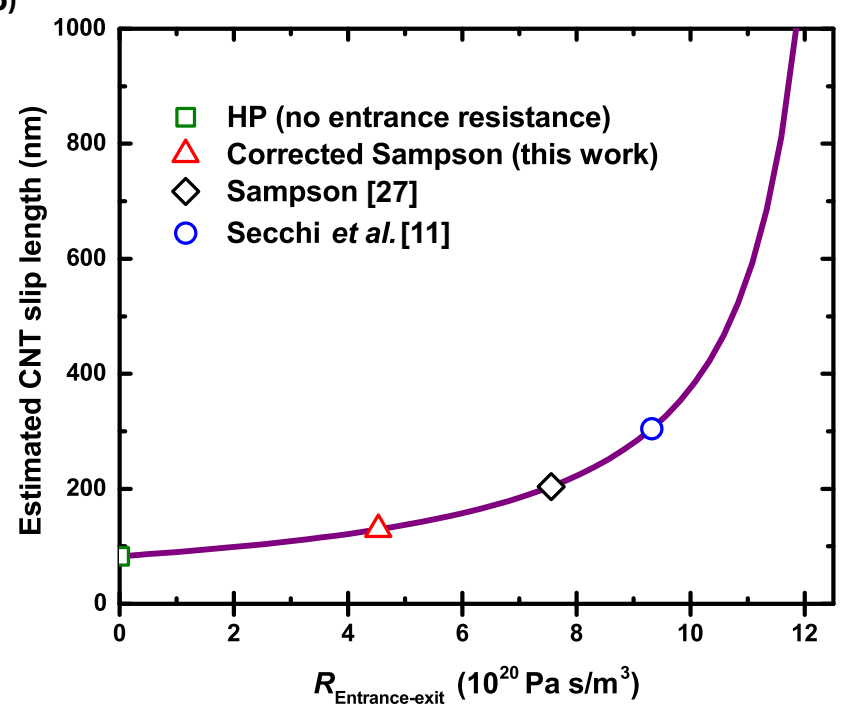

FIG. 6. (a) Comparison of the predicted permeation coefficients from the correct Sampson theory with the experimentally [45-48] and computationally [10,49] measured permeation coefficients. The filled square symbols are the experimental data taken directly from Refs. [45-48], the filled triangle symbols are the MD simulations data from other studies [10,49], and the circle symbols are the predicted permeation coefficients from the corrected Sampson theory. (b) Slip length in a 15-nm-radius CNT (with a length of $700 \mathrm{~nm}$ ), estimated from the experimental total hydrodynamic resistance $R_{\text {total }}$ (or inverse of permeation coefficient) given in Secchi et al. [9], is plotted as a function of varying entrance/exit hydrodynamic resistance $R_{\text {Entrance-exit }}$ (magenta curve). The estimated CNT slip length varies significantly depending on the entrance/exit resistance obtained from different models (symbols). An accurate theory, therefore, is necessary to estimate the slip lengths inside CNTs (or in any other nanotubes) from the experimentally measured permeation coefficients. 
(a)

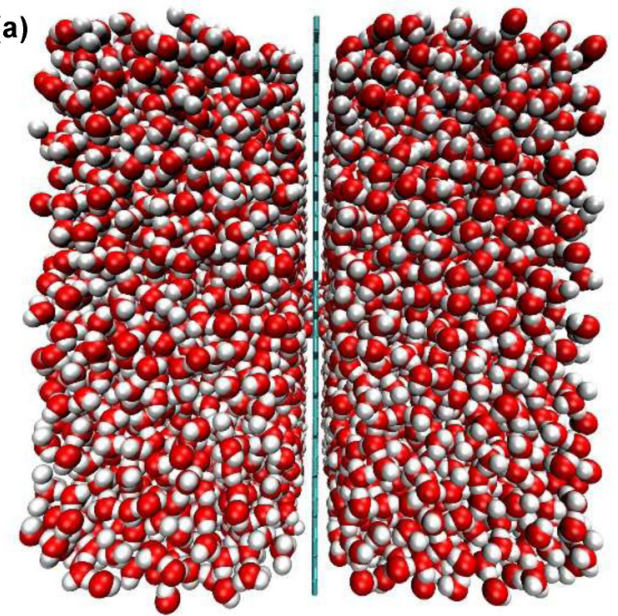

(b)

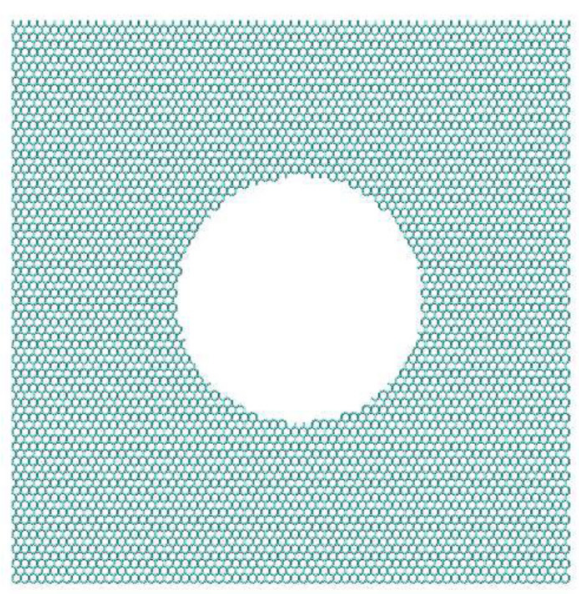

FIG. 7. (a) Simulation box for water across a single-layer graphene nanopore. (b) Membrane view of a nanopore with a radius of $3.54 \mathrm{~nm}$. Oxygen, hydrogen, and carbon atoms are shown in red, white, and cyan, respectively.

to quantitatively describe the flow both inside and outside of the nanotubes. For example, estimation of slip lengths inside CNTs from experimentally measured permeation coefficients (or hydrodynamic resistance) relies on the theories used to obtain the resistance at the ends and inside the CNTs. The total resistance can be written as the sum of the Sampson resistance and HP resistance, $R_{\text {total }}=R_{\text {Entrance-exit }}+R_{\mathrm{CNT}}^{\mathrm{HP}}=$ $\frac{C \mu}{a^{3}}+\frac{8 \mu h}{\pi\left(a^{4}+4 a^{3} \delta \mathrm{CNT}\right)} \cdot R_{\mathrm{total}}$, which is typically obtained from experimental measurements [9], is dominated by $R_{\text {Entrance-exit }}$ and an error in estimation of $R_{\text {Entrance-exit }}$ can result in a significant error in calculation of $R_{\mathrm{CNT}}^{\mathrm{HP}}$, using which the slip length inside the CNT is extracted. $\delta_{\mathrm{CNT}}$ is the slip length inside the CNTs (not at the entrance/exit). $C$ is the prefactor in the Sampson formula $(C=3$ in the original Sampson formula and $C=$ $3\left[\frac{1+2\left(\frac{\alpha}{1+\alpha}\right)^{3 / 2}-3\left(\frac{\alpha}{1+\alpha}\right)}{(1+\alpha)^{3 / 2}}\right]$ for the slip-corrected Sampson formula developed here). In Fig. 6(b), the estimated slip length inside the CNTs $\left(\delta_{\mathrm{CNT}}\right)$ is plotted as a function of different values of entrance/exit resistance (varying the prefactor in the Sampson formula) for one of the experimental data points in Secchi et al. [9] $\left(R_{\mathrm{total}}=12.96 \times 10^{20} \mathrm{~Pa} \mathrm{~s} / \mathrm{m}^{3}\right.$ with a radius of 15 $\mathrm{nm}$ and $h=700 \mathrm{~nm})$. The Sampson formula $(C=3)$ and the continuum fluid mechanics simulations in Secchi et al. [9] (where $C>3$ ) result in large CNT slip lengths of $\sim 204$ and $\sim 300 \mathrm{~nm}$, respectively. However, a value of $C=1.8$ is obtained using the corrected Sampson formula [accessible radius of $a=15-0.17=14.83 \mathrm{~nm}$ with a graphene (entrance/exit) slip length of $\sim 1.5 \mathrm{~nm}$ is assumed]. Hydrodynamic resistance is about $60 \%$ of that of the original Sampson theory as $\frac{a}{\delta}=\frac{1}{\alpha}=9.88$, resulting in a smaller CNT slip length of $129.4 \mathrm{~nm}$ which is much closer to the slip lengths computed from the MD simulations. Therefore, we show that when the entrance/exit resistance is estimated correctly, the experimentally measured total resistance values by Secchi et al. [9] lead to CNT slip lengths consistent with that of MD simulations, and the slip lengths (whether at the entrance/exit or in the middle of the CNTs) calculated by MD simulations are reasonable. The corrected Sampson theory, therefore, could help explain the reported discrepancies in CNT slip lengths between experiments and MD simulations [9].

\section{ACKNOWLEDGMENTS}

This work was supported as part of the Center for Enhanced Nanofluidic Transport (CENT), an Energy Frontier Research Center funded by the U.S. Department of Energy, Office of Science, Basic Energy Sciences under Award No. DE-SC0019112. The simulations were performed using the Extreme Science and Engineering Discovery Environment (XSEDE) [supported by National Science Foundation (NSF) Grant No. OCI1053575] and Blue Waters (supported by NSF Awards No. OCI-0725070, and No. ACI-1238993, and the state of Illinois).

\section{APPENDIX A: MD SIMULATIONS METHODS}

MD simulations were performed using the LAMMPS package [38]. A typical simulation box consists of water molecules and a graphene sheet (see Fig. 7). A nanopore is drilled in a graphene sheet with carbon center-to-center diameters of $0.83,1.10,1.36,1.90,2.44,3.53$, and $7.42 \mathrm{~nm}$. The Cartesian coordinate $(1,2,3)$ is defined such that the $1-2$ plane lies in the plane of the graphene and the 3 axis lies on the $z$ axis in the cylindrical coordinates. The system dimensions vary from 3.75 to $16.3 \mathrm{~nm}$ along the 1 and 2 axes, and from 6 to $12 \mathrm{~nm}$ along the 3 axis depending on the diameter. The dimensions along the 1 and 2 axes change with the radius of the pore to account for the porosity of the nanopore (the ratio of pore area to membrane area is $<0.148$ ). The simulations contain up to $\sim 320000$ atoms. The extended simple point charge (SPC/E) water model was used and the SHAKE algorithm was employed to maintain the rigidity of the water molecule. The carbon-water interactions were modeled by the force-field parameters given in Wu et al. [30]. The carbonwater Lennard-Jones (LJ) parameters were varied to generate different slip lengths. A summary of the MD simulations performed is provided in Table I. Since the carbon atoms are 
TABLE I. Summary of the MD simulations performed in this work. LJ parameter $\varepsilon_{\mathrm{C}-\mathrm{O}}$ (between carbon atom of graphene and oxygen atom of water) is varied for some of the simulations to generate different slip lengths. In the force field by Wu et al. [30], $\varepsilon_{\mathrm{C}-\mathrm{O}}$ is 0.085 $\mathrm{kcal} / \mathrm{mol}$.

\begin{tabular}{lclcc}
\hline \hline $\begin{array}{l}\text { Number of } \\
\text { simulations }\end{array}$ & $\begin{array}{c}\text { Number of C atoms } \\
\text { in each simulation }\end{array}$ & $\begin{array}{c}x, y, \text { and } z \text { dimensions } \\
\text { of simulation box }(\mathrm{nm})\end{array}$ & $a(\mathrm{~nm})$ & $\varepsilon_{\mathrm{C}-\mathrm{O}}(\mathrm{kcal} / \mathrm{mol})$ \\
\hline 2 & 8563 & $3.75,3.75$, and 6 & 0.24 & 0.00001 and 0.0850 \\
2 & 8553 & $3.75,3.75$, and 6 & 0.37 & 0.00001 and 0.0850 \\
2 & 8541 & $3.75,3.75$, and 6 & 0.51 & 0.00001 and 0.0850 \\
1 & 30508 & 5,5, and 12 & 0.78 & 0.0850 \\
1 & 30447 & 5,5, and 12 & 1.05 & 0.0850 \\
1 & 64802 & $7.3,7.3$, and 12 & 1.56 & $0.08501,0.0850,0.1,0.2,0.3,0.4$, \\
11 & 323577 & $16.3,16.3$, and 12 & 3.54 & $0.5,0.6,0.7,0.8$, and 0.9 \\
\hline \hline
\end{tabular}

fixed in space, the interactions between carbon atoms were turned off. The LJ cutoff distance was set to $1.2 \mathrm{~nm}$. The long range electrostatic interactions were calculated by the particle-particle-particle-mesh (PPPM) method [39]. Periodic boundary conditions were applied in all three directions. For each simulation, first the energy of the system was minimized for 10000 steps. Next, the system was equilibrated using a $N P T$ ensemble for $1 \mathrm{~ns}$ at a pressure of $1 \mathrm{~atm}$ (both at the start and the end of the simulations with a damping time of 100 fs) and a temperature of $300 \mathrm{~K}$ with a time step of $1 \mathrm{fs}$. With the graphene atoms held fixed in space, the NPT simulations (the three dimensions are controlled independently using the components of the stress tensor) allow the water to reach its equilibrium density $\left(1 \mathrm{~g} / \mathrm{cm}^{3}\right)$. Then, an additional $N V T$ simulation was performed for $2 \mathrm{~ns}$ to further equilibrate the system. Temperature was maintained at $300 \mathrm{~K}$ by using the NoséHoover thermostat [40,41] with a time constant of 0.1 ps. Finally, the nonequilibrium pressure-driven simulations were carried out in a NVT ensemble for 10 ns to calculate the volumetric flow rates and subsequently obtain the hydrodynamic resistance for the applied pressures. The external pressures were applied using the method described in Refs. [23,42]. External forces are applied on individual oxygen atoms of water within $1 \mathrm{~nm}$ of the end of the simulation box. The applied pressure drop is then obtained from $\Delta P=\frac{f N}{A}$ where $f$ is the individual force on the oxygen atom, $N$ is the number of oxygen atoms within the 1-nm slab region, and $A$ is the area of the cross section (or the graphene membrane). Different pressure drops of 10,50 , and $100 \mathrm{MPa}$ are applied and normalized by their corresponding volumetric flow rates to get the average hydrodynamic resistance (presented as the data points in Fig. 3). Slip lengths [as shown in Fig. 1(a)] are computed from MD simulations [23] using $\delta=\frac{\mu U_{\text {slip }}}{\tau_{\text {interfec }}}$, where $U_{\text {slip }}$ is the slip velocity (which is directly obtained from the velocity profiles), and $\tau_{\text {interface }}$ is the friction force (parallel to the axis of the pore) per unit pore area. The friction force is calculated between the water molecules and the wall atoms.

\section{APPENDIX B: FICTITIOUS HYDROPHILIC MATERIAL WITH A NO-SLIP CONDITION}

As mentioned in the paper, the interaction parameter between the oxygen atom of water and the carbon atom of graphene $\left(\varepsilon_{\mathrm{C}-\mathrm{O}}\right.$, LJ parameter) is increased from the value
$\left(\varepsilon_{\mathrm{C}-\mathrm{O}}=0.085 \mathrm{kcal} / \mathrm{mol}\right)$ given in the force field developed by $\mathrm{Wu}$ et al. [30] until the graphene surface becomes highly hydrophobic and results in a no-slip condition as shown in Fig. 1(b). The different values of $\varepsilon_{\mathrm{C}-\mathrm{O}}$ considered for the 3.54-nm-radius pore (this is a large enough pore where the viscosity in the pore converges to that of the bulk water) are provided in Table I. The velocity profiles for an applied pressure of $50 \mathrm{MPa}$ are shown in Fig. 8. Note that a further increase in the interaction energy leads to negative slip lengths which are not considered in this study. Figure 8(c) shows the density profile of water molecules as a function of the radial distance from the center of the pore. The density profiles are very similar except for their peak (which increases with $\left.\varepsilon_{\mathrm{C}-\mathrm{O}}\right)$ for the different values of $\varepsilon_{\mathrm{C}-\mathrm{O}}$. The volume bins in the simulations are circular shells inside the pore with a thickness of $0.34 \mathrm{~nm}$ (the thickness of the graphene membrane) in the 3 axis (the axis of the pore), and a thickness of 0.2 $\mathrm{nm}$ in the radial direction. The velocity and density profiles are used to calculate the mass flow rates. Volumetric flow rates, $Q$, are then obtained by normalizing the mass flow rates by the bulk water density. Using the volumetric flow rates and the applied pressures, the hydrodynamic resistance values are obtained $\left(R=\frac{\Delta P}{Q}\right)$. The hydrodynamic resistance is calculated to be $5.78 \pm 0.03 \times 10^{22} \mathrm{~Pa} \mathrm{~s} / \mathrm{m}^{3}$ and $4.21 \pm 0.04 \times$ $10^{22} \mathrm{~Pa} \mathrm{~s} / \mathrm{m}^{3}$, for the hydrophilic pore (with no slippage) and the real graphene pore, respectively (the errors are computed based on three different applied pressures of 10, 50, and $100 \mathrm{MPa})$.

\section{APPENDIX C: CONTINUUM SIMULATIONS METHODS}

We performed continuum simulations by numerically solving the steady-state Navier-Stokes (NS) continuity and momentum equations $\left(\nabla \cdot \mathbf{u}=0\right.$ and $\left.\mathbf{u} \cdot \nabla \mathbf{u}=-\frac{\nabla p}{\rho}+v \nabla^{2} \mathbf{u}\right) \cdot p$ is the pressure, and $\rho$ and $v$ are the density and the kinematic viscosity of the fluid, respectively. We solved the NS equations using OPENFOAM V4.1 [43] based on the finite volume method. The values of $\rho$ and $v$ are set to be the bulk values of water $\left(1000 \mathrm{~kg} / \mathrm{m}^{3}\right.$ and $0.85 \times 10^{-6} \mathrm{~m}^{2} / \mathrm{s}$, respectively). We used a 2D domain as shown in Fig. 9 with an unstructured nonuniform mesh with a high resolution around the pore and the membrane walls. The meshing is carried out using GMSH software [44]. The mesh convergence is tested by 

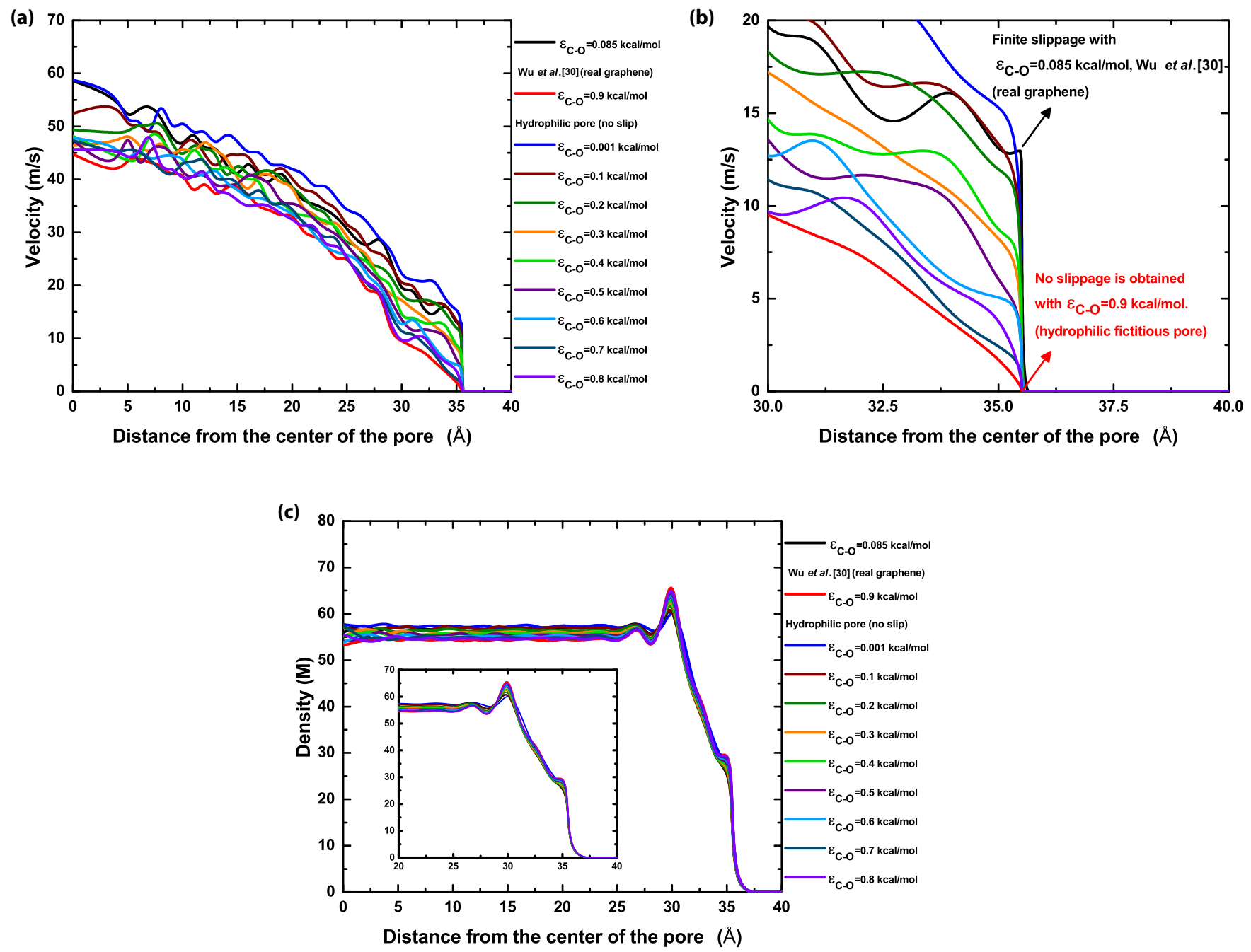

FIG. 8. (a) Axial velocity profile in the single-layer graphene nanopore $(a=3.54 \mathrm{~nm})$ as a function of the radial distance from the center of the pore is plotted for an applied pressure of $50 \mathrm{MPa}$ using the Wu et al. [30] force field (real graphene) and other fictitious force fields (by varying $\left.\varepsilon_{\mathrm{C}-\mathrm{O}}\right)$ until a no-slip condition is achieved $\left(\varepsilon_{\mathrm{C}-\mathrm{O}}=0.9 \mathrm{kcal} / \mathrm{mol}\right)(\mathrm{b}) \mathrm{A}$ zoomed-in part of velocity profile in (a) is presented to clearly show the slippage (in the form of slip velocity) for the real graphene and fictitious hydrophilic pores. (c) Density profile in the single-layer graphene nanopore [for the same systems in $(a, b)]$ as a function of the radial distance from the center of the pore is plotted using different force fields [for the same systems in $(\mathrm{a}, \mathrm{b})$ ]. The unit is in molar concentration (M).

decreasing the mesh size until the change in the maximum velocity between two successive mesh resolutions is $2 \%$. The steady-state solution is reached when the residual error between two successive solutions for velocity and pressure is less than $10^{-6}$. The imposed boundary conditions are summarized in Fig. 9. The thickness of the pore is fixed to $2 \mathrm{~nm}$ in all simulations, except for the velocity profile in Fig. 10 in which we also used $0.34 \mathrm{~nm}$. We used a higher thickness than that of graphene to ensure the velocity is developed inside the pore.

We further obtained the hydrodynamic resistance from the NS continuum simulations for pores with $h=2 \mathrm{~nm}, a=1-5$ $\mathrm{nm}$, and $\Delta P_{o}=20-100 \mathrm{MPa}$ (see Table II for NS simulation details). The slip length, $\delta$, is determined by extrapolating the velocity profiles. The resistance values from NS simulations agree reasonably with those of the slip-corrected Sampson theory (see Fig. 3). Note that since the NS simulations have a uniform viscosity, an appropriate comparison should be made with the slip-corrected Sampson theory (not the viscosity-corrected theory with a two-viscosity model). The small deviation between the resistance values of the NS continuum and the slip-corrected Sampson theory can be related to the following sources of error: (1) continuum simulations at scales of less than $10 \mathrm{~nm}$ require cell size mesh of utmost less than $0.1 \mathrm{~nm}$ which produces larger numerical errors in the spatial discretization; (2) we assumed a perfect slip condition at the side walls of the graphene membrane for all cases for simplicity, whereas it is zero in the Sampson theory; and (3) the thickness of the membrane is $2 \mathrm{~nm}$ in the NS simulations, whereas it is zero in the Sampson theory.

\section{APPENDIX D: COMPARISON OF VELOCITY FIELD FROM MD AND NS CONTINUUM SIMULATIONS WITH THE VELOCITY PROFILES OBTAINED FROM THE CORRECTED SAMPSON THEORY}

A schematic of the velocity profiles inside and outside the orifice based on the corrected Sampson theory is shown in 


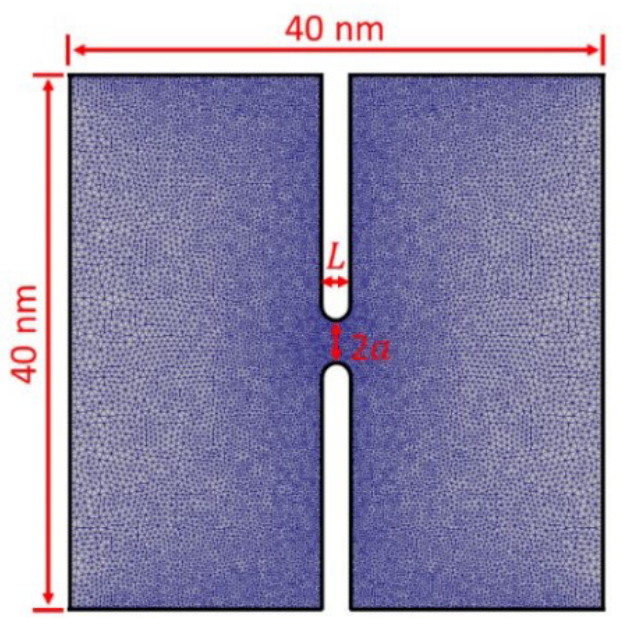

Wall: Perfect slip $\nabla u=0, \nabla p=0$

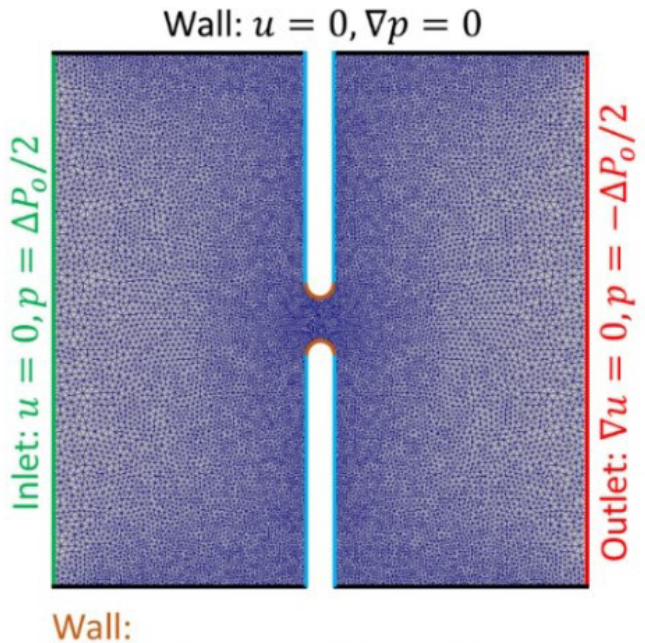

1. No slip: $u=0, \nabla p=0$

2. Partial slip $u=u_{\text {slip }}, \nabla p=0$

3. Perfect slip $\nabla u=0, \nabla p=0$

FIG. 9. The 2D computational domain, mesh, and boundary conditions which are used in the NS continuum simulations. The pressure difference, $\Delta P_{o}$, and the pore radius, $a$, are varied in the simulations. The boundary conditions are indicated in different colors. Inside the pore, we considered three different slip conditions at the wall: no slip, partial slip, and perfect slip. For the partial slip, we imposed a partial velocity, $U_{\text {slip. }}$ The slip length, $\delta$, is then obtained by extrapolating the velocity profile. For simplicity (avoiding singularities in the simulations), we consider a perfect slip condition at the graphene membrane walls (blue boundaries) for all cases.

Fig. 10. Similar to the original Sampson theory, the velocity is set to zero right at the boundaries of the orifice wall inside and outside the pore. Inside the pore, the velocity is only nonzero a distance $d$ away from the orifice boundary based on our proposed stream function in Eq. (6) in the main part of the paper. Outside the pore, $d$ corresponds to $x=b$ (in the oblate spheroidal coordinates) where the velocity is set to zero for

TABLE II. Summary of the NS continuum simulations for a pore with thickness of $2 \mathrm{~nm}$ to obtain the hydrodynamic resistance. Radii, slip lengths, pressure drops, volumetric flow rates, and normalized hydrodynamic resistance are tabulated.

\begin{tabular}{lcccc}
\hline \hline$a(\mathrm{~nm})$ & $\delta(\mathrm{nm})$ & $\Delta P_{o}(\mathrm{MPa})$ & $Q\left(10^{-15} \mathrm{~m}^{3} / \mathrm{s}\right)$ & $R / R^{\text {Sampson }}$ \\
\hline 1 & 0.21 & 50 & 1.23 & 0.707 \\
1.1 & 0.001 & 70 & 1.16 & 1.050 \\
1.15 & 0.01 & 70 & 1.84 & 0.948 \\
1.25 & 0.32 & 50 & 2.01 & 0.433 \\
1.25 & 0.25 & 100 & 2.45 & 0.498 \\
1.5 & 1.13 & 50 & 5.02 & 0.173 \\
1.5 & 0.90 & 50 & 4.36 & 0.199 \\
1.5 & 0.69 & 50 & 3.70 & 0.690 \\
1.5 & 0.50 & 50 & 3.05 & 0.286 \\
2 & 0.28 & 50 & 1.14 & 0.765 \\
2 & 0.20 & 50 & 1.07 & 0.814 \\
2 & 0.33 & 50 & 1.21 & 0.721 \\
2 & 0.41 & 50 & 1.28 & 0.682 \\
2 & 0.50 & 50 & 1.34 & 0.647 \\
5 & 4.50 & 20 & 17.14 & 0.021 \\
5 & 10 & 20 & 9.16 & 0.038 \\
\hline \hline
\end{tabular}

$0<x \leqslant b$ based on the proposed stream function [Eq. (6)]. As shown, this treatment of the velocity field results in a smooth change in the velocity direction along a streamline. As explained in Eqs. (7)-(9), the velocity jump (slip velocity) at a distance $d$ away from the orifice boundary (or when $r=a$, water accessible radius) is used to obtain its corresponding slip length as a function of $d$ and $a$ [Eq. (9)]. As shown in Fig. 10, the treatment used in the corrected Sampson theory results in consistent velocity fields between the corrected theory and the MD-NS simulations. In graphene nanopores, the diameter is larger than its finite thickness $(\sim 0.34 \mathrm{~nm})$ and therefore the flow will not be fully developed to produce a pluglike flow even in the case of perfect slip condition for the NS simulations. To show the velocity profile of different pore thicknesses and the velocity field of different boundary conditions (no slip, partial slip, and perfect slip), we performed steady-state NS simulations. Our continuum simulations show that for the perfect slip condition, the velocity profile in the nanopore with a thickness of $0.34 \mathrm{~nm}$ does not converge to an ideal pluglike profile; however, as we increase the thickness to $2 \mathrm{~nm}$ the velocity profile flattens and approaches the pluglike profile for the same boundary condition [see Fig. 10(c)]. The velocity field profiles for the no-slip, partial slip, and perfect slip conditions for the 2-nm thickness pore are shown in Figs. 10(d)-10(f). In both MD simulations and NS simulations (with a partial slip length), near the graphene surface (inside and outside the pore), the velocity almost vanishes similar to the velocity field in the corrected Sampson theory. The velocity jump [also shown in Fig. 1(b)] inside the pore resembles the velocity jump ( $r=a$, water accessible radius) modeled in the corrected Sampson theory. 
(a)

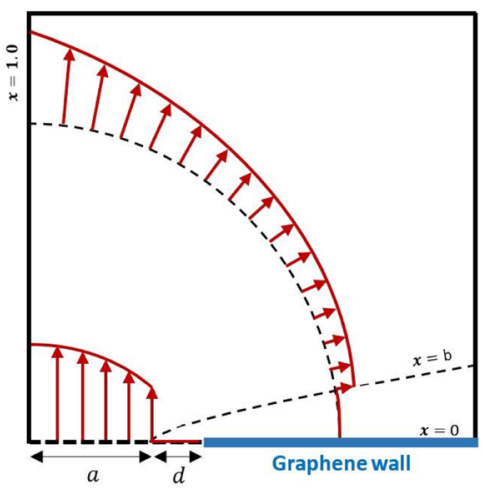

(c)

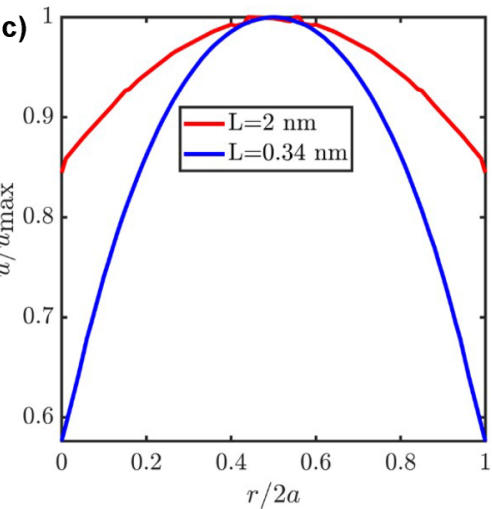

(e)

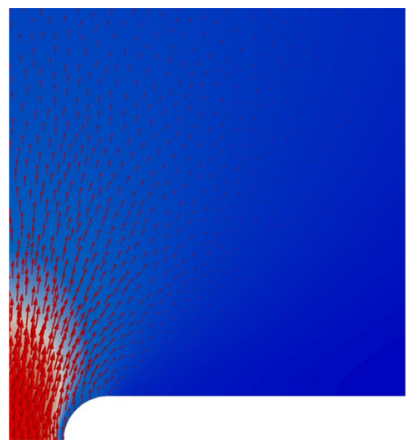

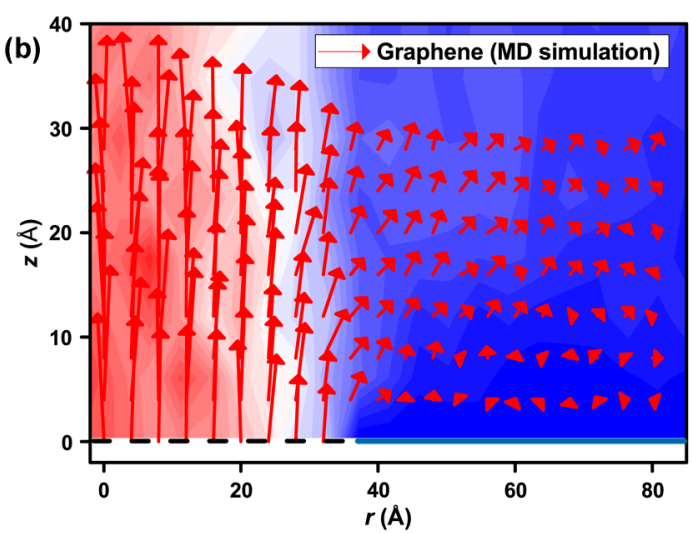

Velocity $(\mathrm{m} / \mathrm{s})$

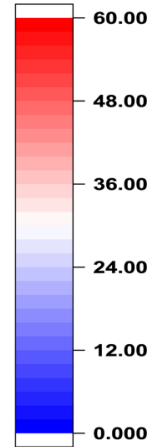

(d)

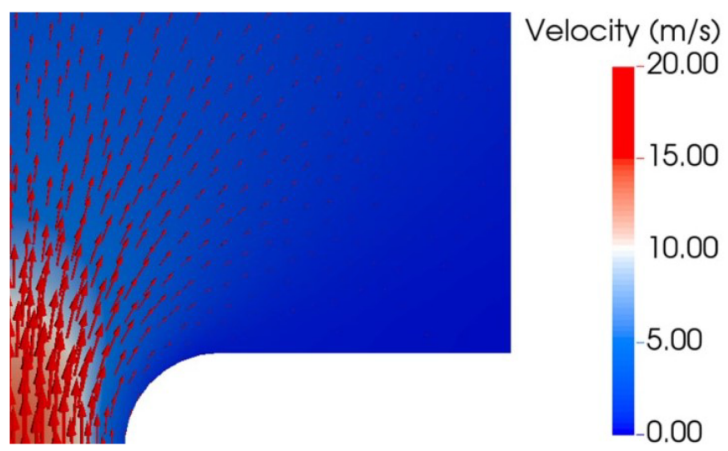

(f)

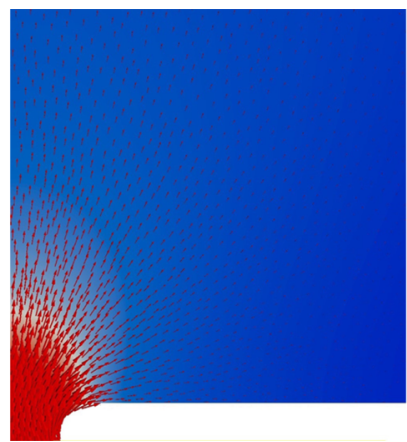

FIG. 10. (a) Schematic of the velocity profiles (red) inside and outside the orifice pore in the corrected Sampson formulation. Inside the orifice, based on the stream function in Eq. (6), the velocity is only nonzero a distance $d$ away from boundary of the orifice (blue). $d$ corresponds to $x=b$ outside the orifice. (b) The velocity vector field (red arrows) is obtained in the MD simulation for a pressure drop of 50 MPa. Near the graphene surface (inside and outside the pore), the velocity almost vanishes similar to the velocity field in the corrected Sampson theory (a). The velocity jump [also shown in Fig. 1(b)] inside the pore resembles the velocity jump ( $r=a$, water accessible radius) modeled in the corrected Sampson theory. Velocity profile and velocity fields: (c)-(f) obtained by NS model for pores with $a=1.2 \mathrm{~nm}$, and pressure difference of $50 \mathrm{MPa}$, (c) Velocity profile, $u$, normalized by the maximum velocity, $u_{\max }$, as a function of the radial distance normalized by the pore diameter for thicknesses of 2 and $0.34 \mathrm{~nm}$. (d)-(f) Velocity field vectors of the pore with thickness of $2 \mathrm{~nm}$ for (d) no-slip, (e) partial slip (slip velocity of $5 \mathrm{~m} / \mathrm{s}$ ), and (f) perfect-slip conditions.

\section{APPENDIX E: OBLATE SPHEROIDAL COORDINATES}

The differential operator in Eq. (4) is given by

$$
D^{2}=\frac{h_{\phi}}{h_{t} h_{x}}\left[\frac{\partial}{\partial t}\left(\frac{h_{x}}{h_{\phi} h_{t}} \frac{\partial}{\partial t}\right)+\frac{\partial}{\partial x}\left(\frac{h_{t}}{h_{\phi} h_{x}} \frac{\partial}{\partial x}\right)\right] .
$$

$h_{x}, h_{t}$, and $h_{\phi}$ are the scale factors in the oblate spheroidal coordinates given by

$$
\begin{aligned}
& h_{x}=\frac{\left[t^{2}+(a+d)^{2} x^{2}\right]^{1 / 2}}{\left(1-x^{2}\right)^{1 / 2}}, h_{t}=\frac{\left[t^{2}+(a+d)^{2} x^{2}\right]^{1 / 2}}{\left[t^{2}+(a+d)^{2}\right]^{1 / 2}}, \\
& h_{\phi}=\frac{\left[t^{2}+(a+d)^{2}\right]^{\frac{1}{2}}}{\left(1-x^{2}\right)^{\frac{1}{2}}} .
\end{aligned}
$$


(a)

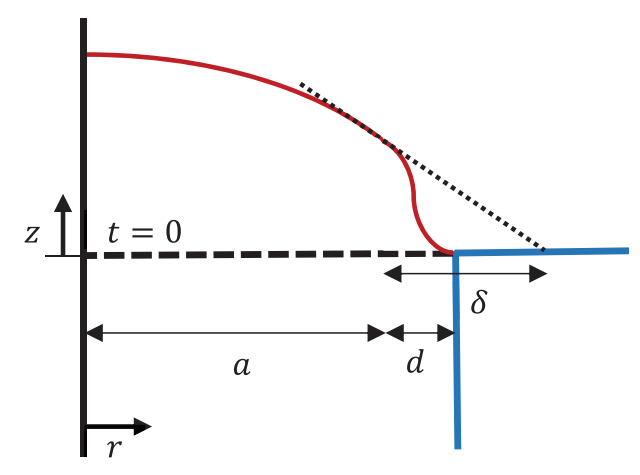

(b)

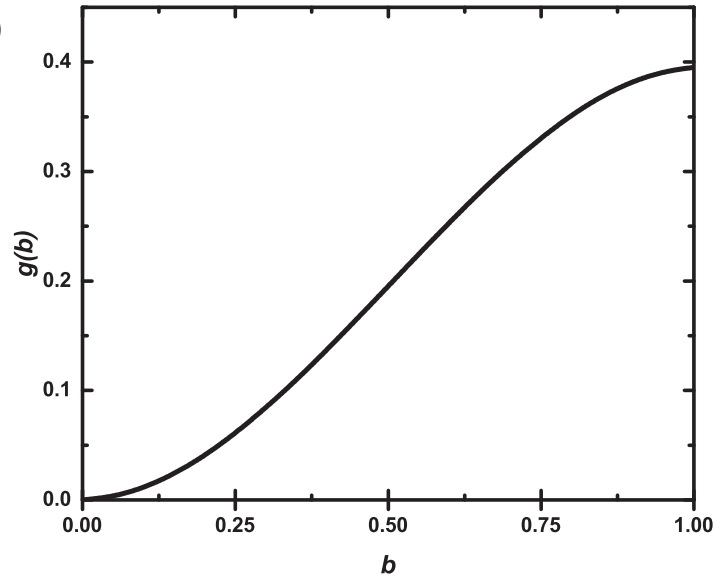

FIG. 11. (a) The schematic of the velocity profile (red curve) and the lengths considered are shown for our trial stream function. The dotted line is the tangent line to the velocity profile at $r=a$ whose intersection with $z=0$ defines the slip length $\delta . d$ is a virtual length within which the velocity decreases rapidly to zero. (b) $g(b)$ is shown for all possible values for $b(0 \leqslant b<1)$. Practically, $g(b)$ is very small for small values of $b$.

\section{APPENDIX F: TRIAL STREAM FUNCTION WITH CONTINUOUS VELOCITY}

As mentioned in the main part of the paper, the proposed stream function has a discontinuity at $x=b$. To overcome the problem of velocity discontinuity, another trial stream function is introduced below:

$$
\psi= \begin{cases}\frac{1}{2 \pi} Q x^{3} & \text { for } b<x \leqslant 1, \\ \frac{1}{2 \pi} Q x^{3}\left(\frac{2 x}{b}-\frac{x^{2}}{b^{2}}\right) & \text { for } 0<x \leqslant b .\end{cases}
$$

Using the above stream function, the velocity (which is obtained from $U_{t}=\frac{1}{r h} \frac{\partial \psi}{\partial x}$ ) remains continuous at $b$. Velocity for $0<x \leqslant b$, which is originally set to zero in the previous stream function in Eq. (6), now decreases rapidly in the depletion and dead zones as shown in Fig. 11. Reperforming the integration in Eq. (5), the hydrodynamic resistance can be obtained from

$$
\begin{aligned}
\frac{\Delta P}{Q}= & \frac{3 \mu}{a^{3}}\left[\frac{1+2\left(\frac{\alpha}{1+\alpha}\right)^{3 / 2}-3\left(\frac{\alpha}{1+\alpha}\right)}{(1+\alpha)^{3 / 2}}\right] \\
& +\frac{3 \mu}{a^{3}}\left[\frac{8}{3(1+\alpha)^{3 / 2}} g(b)\right],
\end{aligned}
$$

where $g(b)=\left(\frac{7}{6} b^{2}-\frac{27}{35} b^{3}\right)$. The second term (which is due to the modified stream function) in the resistance equation can be neglected as it is small compared to the first term. $g(b)$ is plotted in Fig. 11 as a function of $b$, where $g$ is practically very small for small values of $b$. Therefore, for simplicity, one can assume the previous stream function in Eq. (6) and arrive at almost the same hydrodynamic resistance values as obtained from the velocity-continuous stream function proposed above.

\section{APPENDIX G: ESTIMATION OF ACCESSIBLE RADIUS}

As mentioned in the main part of the paper, the water accessible radius $(a)$ is the length within which the water density is nonzero. In Table I, $a$ is calculated based on the water density profiles (the nonzero region excluding the depletion region) from MD simulations. The accessible radius is especially important for small-radius pores. Ideally, however, one should be able to calculate the accessible radius without performing MD simulations. The accessible radius can be systematically obtained by subtracting half of the LJ parameter $\left(\frac{\sigma_{\mathrm{c}-\mathrm{c}}}{2}=0.17 \mathrm{~nm}\right)$ from the carbon center-to-center radius. For the small-radius pores in Table I, the accessible radii are almost identical to the calculated radii using the $\mathrm{LJ}$ parameter (e.g., the calculated radii in the first four rows are 0.2365 , $0.3725,0.508$, and 0.775 , respectively). As shown in Fig. 12,

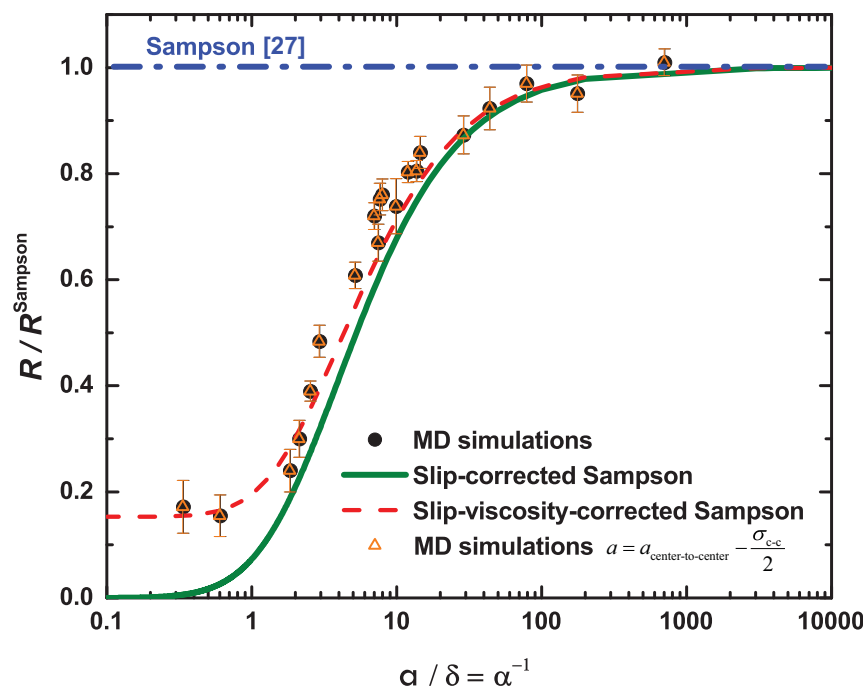

FIG. 12. Figure 3 is replotted with MD simulation data (orange hollow triangles) where the accessible radius $(a)$ is calculated by subtracting half of the LJ parameter $\left(\frac{\sigma_{\mathrm{c}-\mathrm{c}}}{2}=0.17 \mathrm{~nm}\right)$ from the center-to-center radius $\left(a=a_{\text {center-to-center }}-\frac{\sigma_{\mathrm{c}-\mathrm{c}}}{2}\right)$. 
(a)

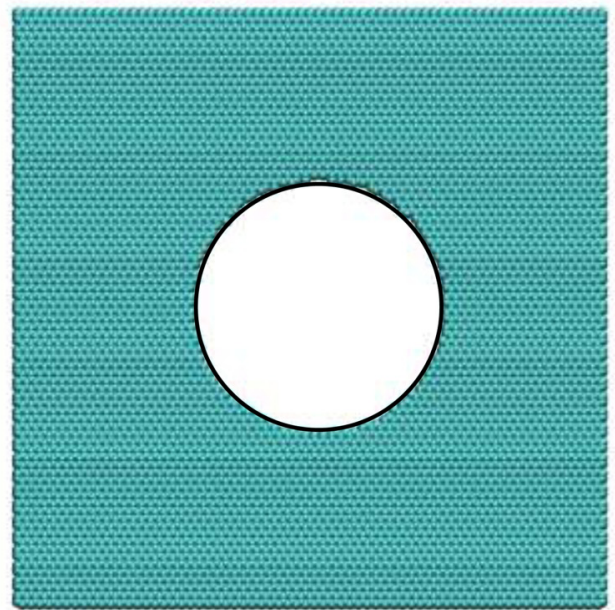

(b)

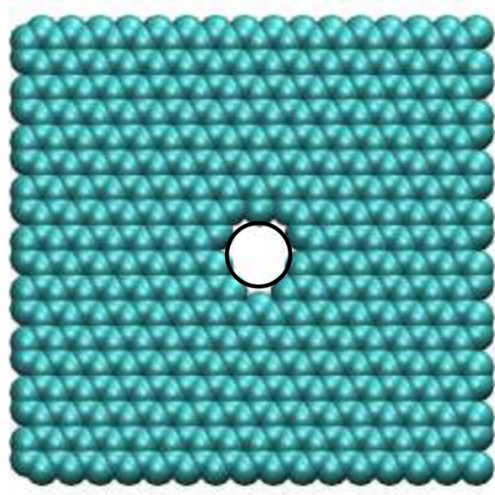

FIG. 13. An overlaid circle is drawn on top of the pores in the largest (a) and smallest (b) pores considered in this work (with a radius of 3.54 and $0.24 \mathrm{~nm}$, respectively). The circles reasonably fit the discrete boundaries of the pores dictated by the carbon atoms at the edge of the pores.

the recalculated data points (orange triangle symbols), based on the calculated accessible radius $a=a_{\text {center-to-center }}-$ $\frac{\sigma_{\mathrm{c}-\mathrm{c}}}{2}$, are on top of the data points (black symbols) where the accessible radius is obtained along the distance within which the water density is zero. Therefore, subtracting $\frac{\sigma_{\mathrm{c}-\mathrm{c}}}{2}=0.17 \mathrm{~nm}$ from the center-to-center radius is a good measure of calculating the accessible radius in graphitic pores.

\section{APPENDIX H: PORE GEOMETRY}

As mentioned in the main part of the paper, our theory assumes circular pores. This assumption was also employed in previous studies [20,21] where the original Sampson theory was used to predict the flow rates in graphene pores. We plotted the largest and smallest pores (with a radius of 3.54 and $0.24 \mathrm{~nm}$, respectively) considered in this work with an overlaid circle as shown in Fig. 13. The van der Waals representation of carbon atoms is used to show the size of carbon clearly. As shown, the assumption that the pore is circular is reasonable even for the smallest pore considered here.

Even for noncircular pores (possibly observed in experiments), one can generally use the hydraulic radius which is the ratio of the cross-sectional area of the pore to the perimeter of the pore. The hydraulic radius allows treating the problems in the same manner as for a circular pore.
[1] K. Liu, J. D. Feng, A. Kis, and A Radenovic, Atomically thin molybdenum disulfide nanopores with high sensitivity for DNA translocation, ACS Nano 8, 2504, (2014).

[2] F. Fornasiero et al., Ion exclusion by sub-2-nm carbon nanotube pores, Proc. Natl. Acad. Sci. USA 105, 17250 (2008).

[3] M. G. Stanford, P. D. Rack, and D. Jariwala, Emerging nanofabrication and quantum confinement techniques for $2 \mathrm{D}$ materials beyond graphene, npj 2D Mater. Appl. 2, 20 (2018).

[4] S. Faucher et al., Critical knowledge gaps in mass transport through single-digit nanopores: A review and perspective, J. Phys. Chem. C 123, 21309 (2019).

[5] J. K Holt et al., Fast mass transport through sub-2-nanometer carbon nanotubes, Science 312, 1034 (2006).

[6] G. Hummer, J. C. Rasaiah, and J. P Noworyta, Water conduction through the hydrophobic channel of a carbon nanotube, Nature 414, 188 (2001).

[7] M. Majumder, N. Chopra, R. Andrews, and B. J Hinds, Nanoscale hydrodynamics-Enhanced flow in carbon nanotubes, Nature 438, 44 (2005).
[8] S. Joseph and N. R. Aluru, Why are carbon nanotubes fast transporters of water? Nano Lett. 8, 452 (2008).

[9] E. Secchi et al., Massive radius-dependent flow slippage in carbon nanotubes, Nature 537, 210 (2016).

[10] M. E. Suk, and N. R. Aluru, Water transport through ultrathin graphene, J. Phys. Chem. Lett. 1, 1590 (2010).

[11] A. B. Farimani, M. Heiranian, and N. R. Aluru, Nano-electromechanical pump: Giant pumping of water in carbon nanotubes, Sci. Rep. 6, 26211 (2016).

[12] K. Kurotobi and Y. Murata, A Single molecule of water encapsulated in fullerene C-60, Science 333, 613 (2011).

[13] A. B. Farimani, Y. B. Wu, and N. R. Aluru, Rotational motion of a single water molecule in a buckyball, Phys. Chem. Chem. Phys. 15, 17993 (2013).

[14] S. Joseph and N. R Aluru, Pumping of Confined Water in Carbon Nanotubes by Rotation-Translation Coupling, Phys. Rev. Lett. 101, 064502 (2008).

[15] M. Heiranian, A. B. Farimani, and N. R. Aluru, Water desalination with a single-layer $\mathrm{MoS}_{2}$ nanopore, Nat. Commun. 6, 8616 (2015). 
[16] H. Li et al., Experimental realization of few layer twodimensional $\mathrm{MoS}_{2}$ membranes of near atomic thickness for high efficiency water desalination, Nano Lett. 19, 5194 (2019).

[17] J. D. Feng et al., Single-layer $\mathrm{MoS}_{2}$ nanopores as nanopower generators, Nature 536, 197 (2016).

[18] S. Gravelle et al., Optimizing water permeability through the hourglass shape of aquaporins, Proc. Natl. Acad. Sci. USA 110, 16367 (2013).

[19] A. B. Farimani, N. R. Aluru, and E Tajkhorshid, Thermodynamic insight into spontaneous hydration and rapid water permeation in aquaporins, Appl. Phys. Lett. 105, 083702 (2014).

[20] J. Buchheim, K. P. Schlichting, R. M. Wyss, and H. G Park, Assessing the thickness-permeation paradigm in nanoporous membranes, ACS Nano 13, 134 (2019).

[21] S. Gravelle, L. Joly, C. Ybert, and L Bocquet, Large permeabilities of hourglass nanopores: From hydrodynamics to single file transport, J. Chem. Phys. 141, 18C526 (2014).

[22] M. E. Suk and N. R. Aluru, Modeling water flow through carbon nanotube membranes with entrance/exit effects, Nanoscale Microscale Thermophys. Eng. 21, 247 (2017).

[23] M. Heiranian and N. R. Aluru, Nanofluidic transport theory with enhancement factors approaching one, ACS Nano 14, 272 (2020).

[24] M. Couette, Studies relating to the motion of liquids, Ann. Chim. Phys. 21, 433 (1890).

[25] G. H. L Hagen, Uber die Bewegung des Wassers in engen cylindrischen Rohren, Poggendorf's Ann. Phys. Chem. 46, 423 (1839).

[26] J. L. M. Poiseuille, Recherches experimentales sur Ie mouvement des liquides dans les tubes de tres petits diametres; 1. Influence de la pression sur la quantite de Iiquide qui traverse les tubes de tres petits diametres, C. R. Acad. Sci. 11, 961 (1840).

[27] R. A. Sampson, On Stokes's current function, Philos. Trans. R. Soc., A 182, 449 (1891).

[28] H. L. Weissberg, End correction for slow viscous flow through long tubes, Phys. Fluids 5, 1033 (1962).

[29] Z. Dagan, S. Weinbaum, and R. Pfeffer, An infinite-series solution for the creeping motion through an orifice of finite length, J. Fluid Mech. 115, 505 (1982).

[30] Y. B. Wu and N. R. Aluru, Graphitic carbon-water nonbonded interaction parameters, J. Phys. Chem. B 117, 8802 (2013).
[31] L. M. Milne-Thomson, Theoretical Hydrodynamics (Dover Publications, New York, 2013).

[32] S. Goldstein and A. R Council, Modern Developments in Fluid Dynamics: An Account of Theory and Experiment Relating to Boundary Layers, Turbulent Motion and Wakes (The Clarendon Press, Oxford, 1938).

[33] J. A. Thomas and A. J. H McGaughey, Reassessing fast water transport through carbon nanotubes, Nano Lett. 8, 2788 (2008).

[34] M. E. Suk and N. R. Aluru, Molecular and continuum hydrodynamics in graphene nanopores, RSC Adv. 3, 9365 (2013).

[35] D. Cohen-Tanugi and J. C. Grossman, Water desalination across nanoporous graphene, Nano Lett. 12, 3602 (2012).

[36] J. D. Feng et al., Identification of single nucleotides in $\mathrm{MoS}_{2}$ nanopores, Nat. Nanotechnol. 10, 1070 (2015).

[37] A. B. Farimani, K. Min, and N. R. Aluru, DNA base detection using a single-layer $\mathrm{MoS}_{2}$, ACS Nano 8, 7914 (2014).

[38] S. Plimpton, Fast parallel algorithms for short-range moleculardynamics, J. Comput. Phys. 117, 1, (1995).

[39] O. Buneman, Computer-simulation using particles (R. W. Hockney and J. W. Eastwood), SIAM Rev. 25, 425 (1983).

[40] S. Nose, A unified formulation of the constant temperature molecular-dynamics methods, J. Chem. Phys. 81, 511 (1984).

[41] W. G. Hoover, Canonical dynamics-equilibrium phase-space distributions, Phys. Rev. A 31, 1695 (1985).

[42] F. Q. Zhu, E. Tajkhorshid, and K. Schulten, Pressure-induced water transport in membrane channels studied by molecular dynamics, Biophys. J. 83, 154 (2002).

[43] http://www.openfoam.com/.

[44] C. Geuzaine and J.-F. Remacle, Gmsh: A 3-D finite element mesh generator with built-in pre- and post-processing facilities, Int. J. Numer. Methods Eng. 79, 1309, (2009).

[45] K. Celebi et al., Ultimate permeation across atomically thin porous graphene, Science 344, 289 (2014).

[46] S. C. O'Hern et al., Selective molecular transport through intrinsic defects in a single layer of CVD graphene, ACS Nano 6 , 10130 (2012).

[47] S. P. Surwade et al., Water desalination using nanoporous single-layer graphene, Nat. Nanotechnol. 10, 459 (2015).

[48] L. Madauss et al., Fabrication of nanoporous graphene/polymer composite membranes, Nanoscale 9, 10487 (2017).

[49] D. Cohen-Tanugi and J. C. Grossman, Water permeability of nanoporous graphene at realistic pressures for reverse osmosis desalination, J. Chem. Phys. 141, 074704 (2014). 